This item was submitted to Loughborough's Research Repository by the author.

Items in Figshare are protected by copyright, with all rights reserved, unless otherwise indicated.

\title{
Indium-mediated 2-oxonia cope rearrangement of 1,4-dienols to 1,3-dienols
}

\section{PLEASE CITE THE PUBLISHED VERSION}

https://doi.org/10.1021/acsomega.8b03118

\section{PUBLISHER}

(c) American Chemical Society

\section{VERSION}

AM (Accepted Manuscript)

\section{PUBLISHER STATEMENT}

This work is made available according to the conditions of the Creative Commons Attribution-NonCommercialNoDerivatives 4.0 International (CC BY-NC-ND 4.0) licence. Full details of this licence are available at: https://creativecommons.org/licenses/by-nc-nd/4.0/

\section{LICENCE}

CC BY-NC-ND 4.0

\section{REPOSITORY RECORD}

Capel, Natalie J., Martin R. Lindley, Gareth J. Pritchard, and Marc C. Kimber. 2019. "Indium-mediated 2oxonia Cope Rearrangement of 1,4-dienols to 1,3-dienols". figshare. https://hdl.handle.net/2134/36715. 
An indium mediated 2-oxonia Cope rearrangement of 1,4-dienols to 1,3-dienols

Natalie J. Capel, ${ }^{a, c}$ Martin R. Lindley, ${ }^{b}$ Gareth J. Pritchard ${ }^{*, a}$ and Marc C. Kimber ${ }^{*, a}$

a School of Science, Department of Chemistry, Loughborough University, Loughborough, Leicestershire, LE11 3TU, UK.

${ }^{b}$ School of Sports Exercise and Health Science, Loughborough University, Loughborough, Leicestershire, LE11 3TU, UK.

${ }^{c}$ (Present address) School of Chemical and Physical Science, Keele University, Staffordshire, ST5 5BG, UK. 


\section{ABSTRACT}

An indium-mediated isomerization of 1,4-dienols to 1,3-dienols is described. This procedure consists of the addition of pentadienylindium, in a protic solvent, to aldehydes giving the kinetic $\mathrm{Y}$-allylation product in high yields. The subsequent conversion of this $\gamma$-allylation product to its thermodynamic 1,3-dienol $\alpha$-isomer can be achieved by its exposure to indium triflate in the presence of a sub-stoichiometric amount of aldehyde at room temperature. This transformation exhibited moderate to good substrate scope and has been shown to proceed by a 2-oxonia Cope rearrangement. 


\section{INTRODUCTION}

1,4-Dienol motifs (1) are a valuable and sought after precursors in organic synthesis due to their unique functional connectivity. 1,4-Dienols 1 can undergo elimination reactions to give trienes such as $\mathbf{2}$ that have been shown to be excellent Diels-Alder platforms (scheme 1), therefore enabling significant molecular complexity to be built up quickly in very few synthetic transformations. ${ }^{1}$ The connectivity of 1,4-dienols (1) has been harnessed in ruthenium catalyzed RCM transformation delivering unique cyclic and polycyclic structures. ${ }^{2}$ Furthermore, 1,4-dienol 1 can undergo facile isomerization to the thermodynamically favored $\alpha$-allylic alcohol product $3(1,3-$ dienol) that is central to numerous natural products of biological importance as well as useful synthetic intermediates $(\text { Scheme } 1)^{3}$

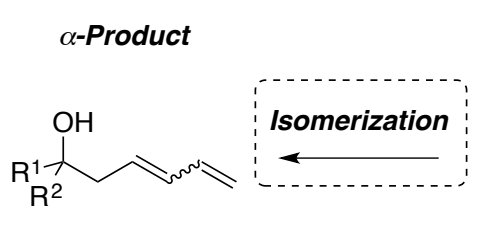

3

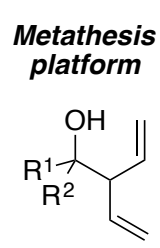

1

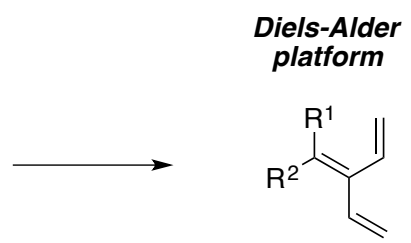

2

Scheme 1. Synthetic utility of 1,4-dienols and their conversion to 1,3-dienols.

The resolvin D- and E-series (Scheme 2, e.g. resolving D1 and resolving E1) are a class of metabolites derived from the $\omega-3$ fatty acids, docosahexaenoic acid (DHA) and eicosapentaenoic acid (EPA). They have been implicated in the regulation of human diseases linked to chronic inflammation such as cardiovascular disease, asthma and rheumatoid arthritis. ${ }^{4 a-c}$ In a project looking at the biological importance of these fatty acids metabolites ${ }^{4 d}$ we required access to a range of racemic 1,3dienols with aryl, alkenyl and alkynyl substitution that mirrored that within the resolvin D1 and E1 structures (scheme 2, 4-6). We envisaged that 1,3-dienols of this type could be accessed via a 2-oxonia Cope rearrangement of a 1,4-dienol, which in turn could be accessed from an appropriate aldehyde (9) and a pentadienyl equivalent (scheme 2). This approach was based on two benchmarks (a) the ability to easily synthesize the 1,4-dienol precursors; and (b) literature precedent for the thermodynamic rearrangement to the required 1,3-dienols. 


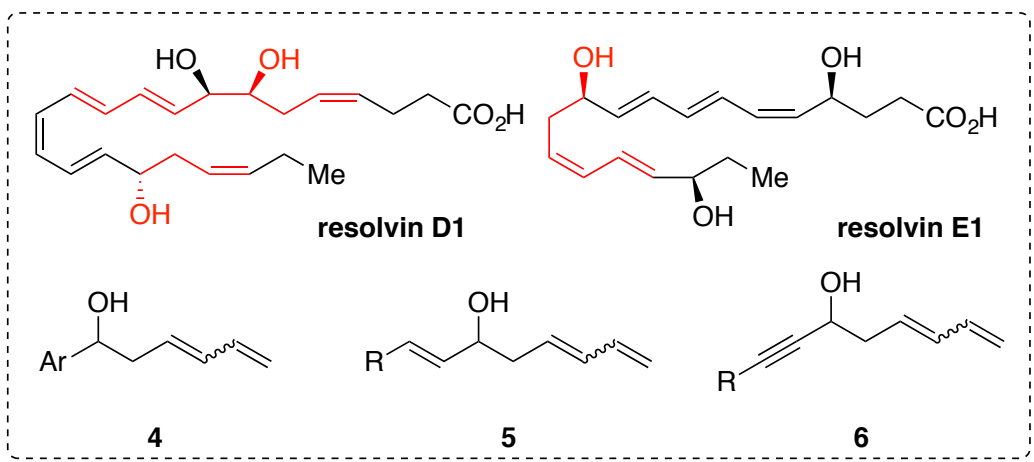

-Nokami3a

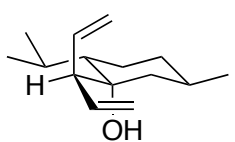

7

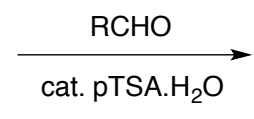

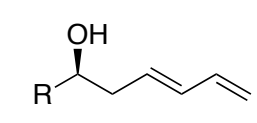

8: $\mathrm{R}$ = aliphatic

-This work

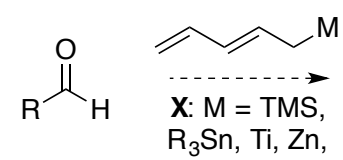

9

In, Br

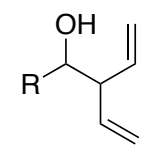

10 2-oxonia Cope<smiles>[R]C(O)CC=CC=C</smiles>

11: $R=$ Aryl, alkenyl, akynyl

Scheme 2. Targeted 1,3-dienols using a 2-oxonia Cope rearrangement.

Firstly, within the literature there are several robust synthetic methods for the synthesis of the 1,4-dienol precursors (10), all of which are based on the addition of a pentadienyl equivalent to a carbonyl substrate 9 (scheme 2 ). These include the Lewis acid mediated addition of 2,4-pentadienyltrimethylsilane ${ }^{5-7}$ and pentadienyltrimethylstannane ${ }^{2 \mathrm{~d}, 8}$ to an aldehyde. Pentadienylzincates ${ }^{9}$ and penta2,4-dienyltitanium complexes ${ }^{10}$ have also been successfully added to carbonyls; and the direct addition of a 1,4-pentadienyl equivalent via treatment with strong base, ${ }^{11}$ has also been successfully employed. An alternate and more environmentally applicable approach has been the addition of pentadienylindium, generated from indium metal and pentadienylbromide, to the requisite carbonyl. Indium has become an important metal in a range Barbier type additions to carbonyls due to its functional group tolerance and low toxicity. ${ }^{12,13}$ Secondly, the 2-oxonia Cope rearrangements are not unprecedented when performed on 1,4-dienols (scheme 2); Nokami ${ }^{3 a}$ and co-workers demonstrated that 1,4-dienol 7 undergoes a diastereoselective, acid catalyzed, 2-oxonia Cope rearrangement to give its a-product 8, in high enantiomeric excess; however, this report was limited to only three aliphatic examples. Therefore, 
we would like to disclose our results on using this 2-oxonia Cope rearrangement to the synthesise 1,3-dienols with aryl, alkenyl and alkynyl substitutions.

\section{RESULTS AND DISCUSSION}

The target compounds for our study necessitated the selection of aldehyde 12 as the starting point. Previous reports had already indicated that DMF, THF and DMSO would be ideal solvents for the addition of bromide 13 , as they all gave the required $\gamma$-addition product. However, in a desire to use more environmentally benign solvents, $\mathrm{H}_{2} \mathrm{O}$ was selected as the starting point, previously used only by Fallis within the context of bromide 13 and a small subset of aldehydes and ketones. ${ }^{1 f, 3 b}$ Consequently, when aldehyde 12 was exposed to 13, indium (1.5 equiv.) and $\mathrm{H}_{2} \mathrm{O}$, it was gratifying to isolate the desired $\gamma$-addition product 14 in $51 \%$ isolated yield (scheme 3). This modest yield and significant amount of unreacted starting material in the crude ${ }^{1} \mathrm{H}$ NMR led us to question the solubility of starting material 12 in $\mathrm{H}_{2} \mathrm{O}$ as solvent. To address this a 3:1 mixture of ethanol ${ }^{14}$ and water was trialed leading to a welcomed increase isolated yield of $67 \%$ confirming our speculation. Finally, further increase in isolated yield to $81 \%$ was obtained when absolute ethanol was used.

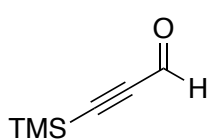

12

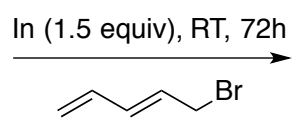

13

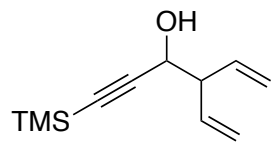

14 $\mathrm{H}_{2} \mathrm{O}, 51 \%$ EtOH: $\mathrm{H}_{2} \mathrm{O}(3: 1), 67 \%$ EtOH, $81 \%$

Scheme 3. Indium mediated addition of bromide 13 to aldehyde 12 in $\mathrm{H}_{2} \mathrm{O}$, ethanol and mixtures thereof.

With optimized conditions in hand, we then examined these conditions on range of aryl, alkenyl and alkynyl aldehydes that were appropriate for our study (scheme 4). 


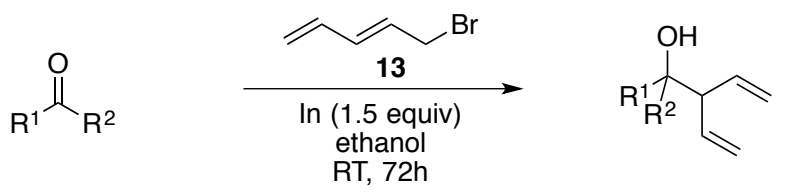

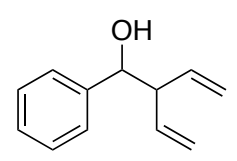

$15,92 \%$<smiles>C=CC(C=C)C(O)c1ccc(Br)cc1</smiles>

18, $90 \%$

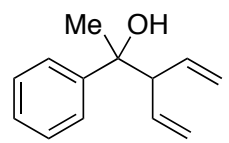

21a, $76 \%$

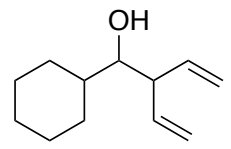

23, $76 \%$<smiles>C=CC(C=C)C(O)c1ccccc1Br</smiles>

$16,89 \%$<smiles>C=CC(C=C)C(O)c1ccc(OC)cc1</smiles>

$19,5 \%$<smiles>C=C/C=C/CC(C)(O)c1ccccc1</smiles>

21b, $>5 \%$<smiles>C=CC(C=C)C(O)CC(C)C</smiles>

24, $69 \%$<smiles>C=CC(C=C)C(O)c1cccc(Br)c1</smiles>

17, $71 \%$<smiles>C=CC(C=C)C(O)c1cccc2ccccc12</smiles>

$20,86 \%$<smiles>C=CC(C=C)C(O)/C=C/c1ccccc1</smiles>

22, $60 \%$<smiles>C=CC(C=C)C(O)C#CS(C)(C)C</smiles>

$14,80 \%$ $12 \mathrm{mmol}$ scale<smiles>C=CC(C=C)C(O)CO</smiles>

$25,72 \%$<smiles>OC1COC(O)CO1</smiles>

26

Scheme 4. Synthesis of 1,4-dienols.

Benzaldehyde and 2-, 3-, and 4-bromobenzaldehyde gave the expected 1,4-dieneol products (15-18) in good to excellent yields of 71-92\%; however, $p$-anisaldehyde yielded its addition product 19 in a very poor isolated yield of only $5 \%$, due likely to the deactivation of the electrophile by the methoxy group. An increase in steric bulk was accommodated as indicated by the successful conversion of $\alpha$-naphthaldehyde giving its addition product $\mathbf{2 0}$ in $\mathbf{8 6 \%}$ isolated yield. Acetophenone also underwent smooth addition to give the $y$-addition product 21a in 76\%; however, within the crude ${ }^{1} \mathrm{H}$ NMR, a small trace amount of the $\alpha$-addition product $\mathbf{2 2} \mathbf{b}$ could be identified, possibly due to the increase in steric bulk of the electrophile. Cinnamaldehyde gave 
its addition product $\mathbf{2 2}$ in $60 \%$ isolated yield, and both linear and branched aliphatic aldehydes gave the desired 1,4-dienols in good to high yields giving 23 and 24 in $76 \%$ and $69 \%$ yields, respectively. The alkynyl aldehyde 12 , crucial to our own work, ${ }^{4 \mathrm{~d}}$ could also be scaled up to $12 \mathrm{mmol}$, giving the addition product 14 in an isolated yield of $80 \%$. We also found that glycoaldehyde dimer $\mathbf{2 6}$ could be utilized in this process giving access to the diol 25 in $72 \%$ isolated yield.

The isomerization of the 1,4-dienols to the desired 1,3-dienols was optimized using substrate 15 (scheme 5 and table 1).

Scheme 5 and table 1. Optimization experiments for the conversion of 1,4-dienol 15 to its respective conjugated species $27 .^{a}$

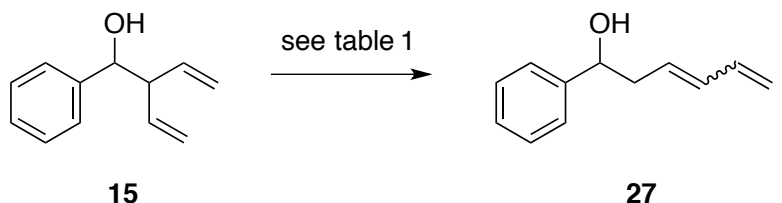

\begin{tabular}{|c|c|c|c|c|}
\hline entry & Lewis Acid $^{b}$ & $\begin{array}{c}\text { Benzaldehyde } \\
(\mathrm{mol} \%)\end{array}$ & Temp. $\left({ }^{\circ} \mathrm{C}\right)$ & Yield $27[\%]^{c}$ \\
\hline 1 & - & - & $\mathrm{rt}$ & - \\
\hline 2 & - & - & 40 & - \\
\hline 3 & $\ln (\mathrm{OTf})_{3}$ & - & $\mathrm{rt}$ & 23 \\
\hline 4 & $\mathrm{AlCl}_{3}$ & - & $\mathrm{rt}$ & 22 \\
\hline 5 & $\mathrm{Sn}(\mathrm{OTf})_{2}$ & - & $\mathrm{rt}$ & 23 \\
\hline 6 & $\ln (\mathrm{OTf})_{3}$ & 10 & $\mathrm{rt}$ & 67 \\
\hline 7 & $\ln (\mathrm{OTf})_{3}$ & 20 & $\mathrm{rt}$ & 65 \\
\hline 8 & $\ln (\mathrm{OTf})_{3}$ & 50 & $\mathrm{rt}$ & 67 \\
\hline 9 & $\ln (\mathrm{OTf})_{3}$ & 100 & $\mathrm{rt}$ & 67 \\
\hline 10 & - & 10 & $\mathrm{rt}$ & - \\
\hline 11 & $\ln (\mathrm{OTf})_{3}$ & 10 & 40 & 63 \\
\hline 12 & $\ln (\mathrm{OTf})_{3}$ & - & 40 & 19 \\
\hline $13^{d}$ & $\ln (\mathrm{OTf})_{3}$ & 10 & $\mathrm{rt}$ & 66 \\
\hline
\end{tabular}

${ }^{a}$ Reactions were performed in $\mathrm{CH}_{2} \mathrm{Cl}_{2}$ under $\mathrm{N}_{2}$ atmosphere at $0.5 \mathrm{M}$ for $24 \mathrm{~h}$ unless otherwise stated. ${ }^{b} 10 \mathrm{~mol} \%$ of Lewis acid. ${ }^{c}$ Isolated yields in all cases. ${ }^{d} 48 \mathrm{~h}$ reaction time. 
Initially, we found that simple heating of 15 did not yield the desired 1,3-dienol but simply returned starting material (entries 1 and 2). We then investigated the use of a Lewis acid catalyst to effect the rearrangement of 15 to 27 and found that $\ln (\mathrm{OTf})_{3},{ }^{15 a}$ $\mathrm{Sn}(\mathrm{OTf})_{2}$, and $\mathrm{AlCl}_{3}$, all at room temperature, achieved the rearrangement, but in poor isolated yields (entries 3-5). ${ }^{16}$ It must be noted that 27 was isolated in a $E: Z$ ratio of approx. 9:1, in favor of the E-stereoisomer, in each case. With these promising results in hand, we next examined the addition of benzaldehyde in an effort to promote the 2-oxonia Cope rearrangement. ${ }^{3 a, 15 b, c}$ Accordingly, upon the addition of $10 \mathrm{~mol} \%$ of benzaldehyde, in the presence of $\ln (\mathrm{OTf})_{3}$ we isolated the desired 1,3-diene-ol 27 in a dramatically improved yield of 67\% (entry 6). A screen in the amount of benzaldehyde was then examined but no discernible increase in isolated yields was observed (entries 7-9). A control experiment in the absence of Lewis acid, but in the presence of $10 \mathrm{~mol} \%$ of benzaldehyde, confirmed the crucial role of the Lewis acid in this rearrangement (entry 10). An increase in reaction temperature had no significant effect on the isolated product yield of 27 (entry 11); and when this elevated temperature attempt was performed in the absence of benzaldehyde (entry 12) the result mirrored that in entry 3 . Finally, extending the reaction time had no noticeable effect in the isolated yield of 27 (entry 13).

With conditions for the 2-oxonia Cope rearrangement of 1,4-dienol 15 to 27 now identified, the scope of this reaction process was examined using the 1,4-dienols from scheme 4 (scheme 6). 


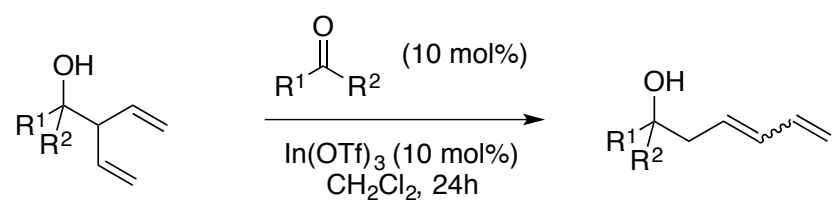

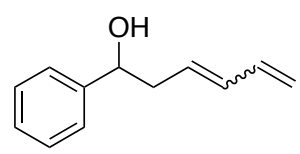

27, $67 \%$ (5 mmol scale) $E / Z 87: 13$<smiles>C=CC=CCC(O)c1ccc(Br)cc1</smiles>

$30,72 \%$
$E / Z 9: 1$

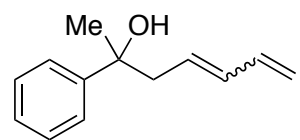

21b, $0 \%$

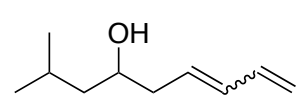

$35,63 \%$ E/Z 76:24

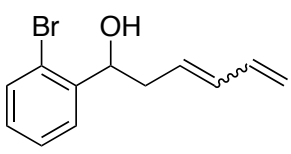

28, $71 \%$

E/Z 99:1<smiles>C=CC=CCC(O)c1ccc(OC)cc1</smiles>

31, $0 \%$<smiles>C=CC=CCC(O)C=Cc1ccccc1</smiles>

33, $0 \%$

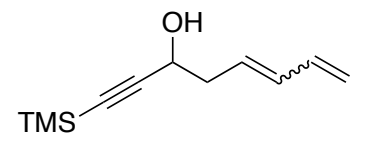

36, $53 \%$ $E / Z 9: 1$<smiles>C=C/C=C/CC(O)c1cccc(Br)c1</smiles>

29, $69 \%$ $E / Z 88.12$<smiles>C=CC=CCC(O)c1cccc2ccccc12</smiles>

$32,72 \%$
$E / Z 87: 13$<smiles>C=CC=CCC(O)C1CCCCC1</smiles>

34, $60 \%$ E/Z3:2<smiles>C=CC=CCC(O)CO</smiles>

$37,>5 \%$

Scheme 6. Synthesis of 1,3-dienols.

This process was met with moderate success. The 2-, 3-, and 4-bromoaryl analogues were all rearranged to the target 1,3-diene-ol in good isolated yields giving 28, 29 and 30, respectively. The a-naphthyl analogue participated in the rearrangement giving the thermodynamic product 32 in good yield, and the rearrangement was tolerant of alkyl substitution as exemplified by the cyclohexyl 34 and isovaleryl 35 examples. The aryl 1,4-diene 15 could be rearranged at an increased scale of $5 \mathrm{mmol}$ giving the 1,3-diene 27 in comparable chemical yield. Finally, the TMS protected alkynyl-1,4-diene 14 was converted to its 1,3-diene isomer 36 in a good yield, and pleasingly this gave a valuable building block that could be potentially used in further work towards the synthesis of the resolvins and other marine derived fatty acids. Unfortunately, the 4-methoxyaryl (22), pent-4-en-2ol (21a), cinnammyl (22) and 1,2-diol 25 precursors, gave little or none of the desired 
products $(31,33,21 \mathrm{~b}$ and 37 , respectively), as well as yielding complex mixtures of products as identified by NMR in each case. With regard to the $E / Z$ stereoisomer ratio, the majority of the aryl derivatives were obtained in a ratio in-line with the parent phenyl analogue, i.e. E/Z ratio of approx. 9:1. However, the 2-bromophenyl derivative, 30, was obtained as the E-isomer exclusively, with none of its Z-isomer observed in the ${ }^{1} \mathrm{H}$ NMR spectra. In contrast, the cyclohexyl (30) and isovaleryl (31) analogues were obtained in $E / Z$ ratios of $3: 2$ and 3:1, respectively.

The 1,3-dienol 37 derived from 1,2-diol 25 would have contained a useful synthetic handle; however, its failure to participate in the rearrangement could be mitigated as shown below in scheme 7 . Treatment of 15 with ethyl propiolate gave the enol ester 38, that when treated with TFA gave the thermodynamic diene 39 in $82 \%$ in an $E / Z$ ratio of 94:6, and with a 1,3-diene with a useful synthetic handle for further manipulation.

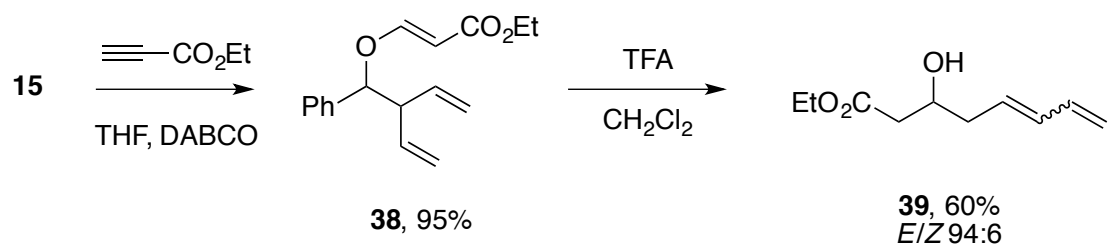

Scheme 7. Acid catalyzed rearrangement of 1,2-diol 38.

Mechanistically the 2-oxonia Cope rearrangement follows the reports previously published (scheme 5). ${ }^{3,15}$ Firstly, Lewis acid activation of benzaldehyde promotes formation of the oxocarbenium ion 41 via 40 . This oxocarbenium ion 41 then undergoes a 2-oxonia Cope rearrangement to deliver the thermodynamic product $\mathbf{4 2}$, with none of the Prins cyclisation product $\mathbf{4 3}$ being detected or isolated. It is assumed $^{15,17}$ that the 2-oxonia Cope rearrangement goes via the corresponding chair transition states. The dominant stereoisomer in each rearrangement was of $E$ stereochemistry, however in some cases a small amount of the Z-stereoisomer could be detected by ${ }^{1} \mathrm{H}$ NMR spectroscopy, and this presumably occurs via bond rotation between C2-C3 within 41 as shown by 41a and 41aa (scheme 7). 


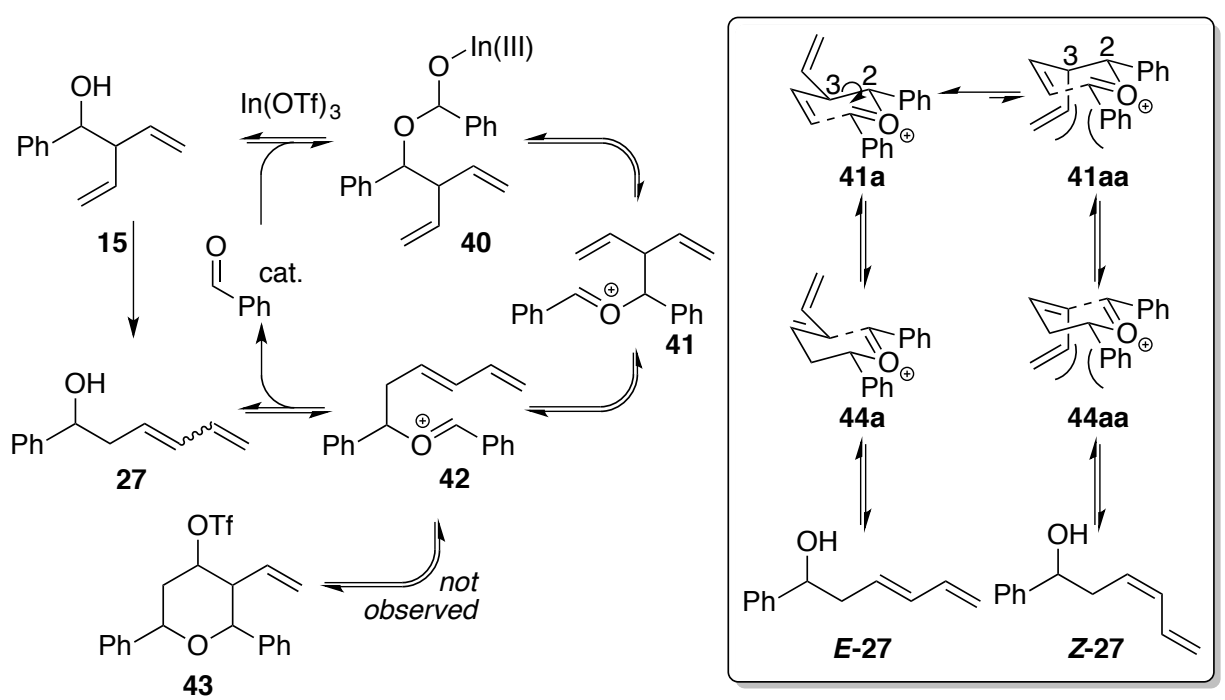

Scheme 7. Mechanism for the kinetic to thermodynamic isomerization using $\ln (\mathrm{OTf})_{3}$.

\section{CONCLUSIONS}

In summary, we have reported a two-step process for the synthesis of 1,3-dienols. A 2-oxonia Cope rearrangement was utilized to rearrange 1,4-dienols to their thermodynamic 1,3-dienol products, and this was facilitated by Lewis acid catalysis with a sub-stoichiometric amount of the requisite aldehyde. This process has modest substrate scope, but unfortunately failed with cinnamyl-substituted systems. However, it was demonstrated to be tolerant of alkynes, which in the context of our research programme should provide a suitable synthetic building for synthesizing fatty acid analogues.

\section{EXPERIMENTAL PROCEDURES}

\section{General procedure for indium addition with (E)-5-bromopenta-1,3-diene.}

Aldehyde or ketone $(1.00 \mathrm{mmol})$ and $(E)$-5-bromopenta-1,3-diene 13 (1.20 mmol) were added to absolute ethanol $(6 \mathrm{~mL})$ and allowed to stir at room temperature. Indium powder (1.50 mmol) was added slowly, so as not to increase the temperature of the reaction mixture, and then allowed to stir at room temperature for $72 \mathrm{~h}$. The reaction mixture was diluted with diethyl ether and then filtered through a plug of silica. The solvent was then removed by evaporation under reduced pressure and the crude product purified by column chromatography. 
1-Phenyl-2-vinylbut-3-en-1-ol (15). ${ }^{8 \mathrm{~d}}$ The title compound was isolated by column chromatography $\left(4: 1\right.$, light petroleum: ethyl acetate, $\left.R_{f}=0.4\right)$ yielding a colorless liquid (157 mg, $0.90 \mathrm{mmol}, 92 \%):{ }^{1} \mathrm{H} \mathrm{NMR}\left(\mathrm{CDCl}_{3}, 400 \mathrm{MHz}\right): \delta 7.41-7.28(\mathrm{~m}, 5 \mathrm{H})$, 5.88 (ddd, $J=17.2,10.4,8.2 \mathrm{~Hz}, 1 \mathrm{H}$ ), 5.71 (ddd, $J=17.2,10.4,7.2 \mathrm{~Hz}, 1 \mathrm{H}$ ), 5.26 (dt, $J=9.6,0.8 \mathrm{~Hz}, 1 \mathrm{H}), 5.22(\mathrm{dt}, J=16.0,1.2 \mathrm{~Hz}, 1 \mathrm{H}), 5.07$ (dt, $J=10.4,1.2 \mathrm{~Hz}$, $1 \mathrm{H}), 5.04(\mathrm{dt}, J=17.2,1.2 \mathrm{~Hz}, 1 \mathrm{H}), 4.61(\mathrm{dd}, J=6.8,2.0 \mathrm{~Hz}, 1 \mathrm{H}), 3.13(\mathrm{dq}, J=7.2$, $0.8 \mathrm{~Hz}, 1 \mathrm{H}), 2.27$ (br s, $1 \mathrm{H}) ;{ }^{13} \mathrm{C}$ NMR $\left(\mathrm{CDCl}_{3}, 100 \mathrm{MHz}\right) \delta 140.8,135.8,135.7$, 127.1, 126.9, 125.7, 117.4, 116.1, 75.2, 55.2; IR (NaCl): 3079, 3066, 1634, $1603 \mathrm{~cm}^{-}$ 1; HRMS (ESI): $m / z[M+H]^{+}$calcd for $\mathrm{C}_{12} \mathrm{H}_{14} \mathrm{O}: 175.1123$, found: 175.1126 .

1-(2-Bromophenyl)-2-vinylbut-3-en-1-ol (16). The title compound was isolated by column chromatography ( $4: 1$, light petroleum: ethyl acetate, $\left.R_{f}=0.4\right)$ yielding a colorless liquid (225 mg, $0.89 \mathrm{mmol}, 89 \%):{ }^{1} \mathrm{H} \mathrm{NMR}\left(\mathrm{CDCl}_{3}, 400 \mathrm{MHz}\right) \delta 7.50$ (dd, $\mathrm{J}=$ 8.0, 1.2 Hz, 1H), $7.46(\mathrm{~d}, J=7.6 \mathrm{~Hz}, 1 \mathrm{H}), 7.31(\mathrm{t}, J=15.2 \mathrm{~Hz}, 1 \mathrm{H}), 7.11(\mathrm{dt}, J=7.6$, $2.0 \mathrm{~Hz}, 1 \mathrm{H}), 5.95-5.83(\mathrm{~m}, 2 \mathrm{H}), 5.21-5.01(\mathrm{~m}, 5 \mathrm{H}), 3.20(\mathrm{q}, J=6.8 \mathrm{~Hz}, 1 \mathrm{H}), 2.25$ $(\mathrm{s}, 1 \mathrm{H}) ;{ }^{13} \mathrm{C} \mathrm{NMR}\left(\mathrm{CDCl}_{3}, 100 \mathrm{MHz}\right) \delta 141.1,137.1,135.6,132.7,128.9,129.6$, 127.7, 122.6, 118.6, 117.0, 74.5, 54.5; IR (NaCl): 3427, 3077, 1636, $619 \mathrm{~cm}^{-1}$; HRMS (ESI): $m / z[M+H]^{+}$calcd for $\mathrm{C}_{12} \mathrm{H}_{12} \mathrm{O}^{79} \mathrm{Br}: 251.0066$, found: 251.0068 .

1-(3-Bromophenyl)-2-vinylbut-3-en-1-ol (17). The title compound was isolated by column chromatography $\left(4: 1\right.$, light petroleum: ethyl acetate, $\left.R_{f}=0.4\right)$ yielding a colorless liquid (180 mg, $0.71 \mathrm{mmol}, 71 \%):{ }^{1} \mathrm{H}$ NMR $\left(\mathrm{CDCl}_{3}, 400 \mathrm{MHz}\right) \delta 7.51-7.49$ $(\mathrm{m}, 1 \mathrm{H}), 7.41(\mathrm{dt}, J=6.8,2.0 \mathrm{~Hz}, 1 \mathrm{H}), 7.26-7.19(\mathrm{~m}, 2 \mathrm{H}), 5.83$ (ddd, $J=17.2,10.4$, $8.0 \mathrm{~Hz}, 1 \mathrm{H}$ ), 5.69 (ddd, $J=17.6,10.4,7.2 \mathrm{~Hz}, 1 \mathrm{H}$ ), 5.27 (ddd, $J=10.4,1.6,0.8 \mathrm{~Hz}$, $1 \mathrm{H}), 5.18(\mathrm{dd}, J=17.2,1.6,0.8 \mathrm{~Hz}, 1 \mathrm{H}), 5.10(\mathrm{dt}, J=10.4 .1 .2 \mathrm{~Hz}, 1 \mathrm{H}), 5.04(\mathrm{dt}, J=$ 17.2, $1.2 \mathrm{~Hz}, 1 \mathrm{H}), 4.56(\mathrm{~d}, J=6.8 \mathrm{~Hz}, 1 \mathrm{H}), 3.07$ (q, $J=7.2 \mathrm{~Hz}, 1 \mathrm{H}), 2.40$ (bs, $1 \mathrm{H}$ ); ${ }^{13} \mathrm{C} \mathrm{NMR}\left(\mathrm{CDCl}_{3}, 100 \mathrm{MHz}\right) \delta 144.2,136.3,136.2,130.7,129.9,129.7,125.6,118.8$, 117.6, 122.3, 75.5, 56.2; IR (NaCl): 3400, 3084, 1637, $739 \mathrm{~cm}^{-1}$; HRMS (ESI): $\mathrm{m} / \mathrm{z}$ $[\mathrm{M}+\mathrm{H}]^{+}$calcd for $\mathrm{C}_{12} \mathrm{H}_{12} \mathrm{O}^{79} \mathrm{Br}: 251.0066$, found: 251.0058 .

1-(4-Bromophenyl)-2-vinylbut-3-en-1-ol (18). ${ }^{\text {1a }}$ The title compound was isolated by column chromatography ( $4: 1$, light petroleum: ethyl acetate, $\left.R_{f}=0.4\right)$ yielding a colorless liquid (228 mg, $0.90 \mathrm{mmol}, 90 \%):{ }^{1} \mathrm{H} \mathrm{NMR}\left(\mathrm{CDCl}_{3}, 400 \mathrm{MHz}\right) \delta 7.44$ (d, J = $8.0 \mathrm{~Hz}, 2 \mathrm{H}$ ), 7.17 (d, J = 8.4 Hz, 2H), 5.80 (ddd, $J=18.0,10.8,8.4 \mathrm{~Hz}, 1 \mathrm{H}), 5.64$ (ddd, $J=17.6,10.8,7.2 \mathrm{~Hz}, 1 \mathrm{H}), 5.25-5.13(\mathrm{~m}, 2 \mathrm{H}), 5.07-4.97(\mathrm{~m}, 2 \mathrm{H}), 4.54$ (dd, $J$ 
$=7.2,3.2 \mathrm{~Hz}, 1 \mathrm{H}), 3.03(\mathrm{q}, J=7.6 \mathrm{~Hz}, 1 \mathrm{H}), 2.24(\mathrm{~d}, J=3.2 \mathrm{~Hz}, 1 \mathrm{H}) ;{ }^{13} \mathrm{C} \mathrm{NMR}$ $\left(\mathrm{CDCl}_{3}, 100 \mathrm{MHz}\right) \delta 140.8,136.4,136.3,131.2,128.6,121.5,118.8,117.6,75.5$, 56.3; IR (NaCl): 3420, 3079, 1635, $623 \mathrm{~cm}^{-1}$; HRMS (ESI): $\mathrm{m} / \mathrm{z}[\mathrm{M}+\mathrm{H}]^{+}$calcd for $\mathrm{C}_{12} \mathrm{H}_{12} \mathrm{O}^{79} \mathrm{Br}: 251.0066$, found: 251.0068 .

1-(4-Methoxyphenyl)-2-vinylbut-3-en-1-ol (19)..$^{\text {gb }}$ The title compound was isolated by column chromatography ( $4: 1$, light petroleum: ethyl acetate, $\left.R_{f}=0.3\right)$ yielding a dark yellow liquid (11 mg, $0.05 \mathrm{mmol}, 5 \%):{ }^{1} \mathrm{H} \mathrm{NMR}\left(\mathrm{CDCl}_{3}, 400 \mathrm{MHz}\right) \delta 7.22(\mathrm{~d}, J=$ $8.0 \mathrm{~Hz}, 2 \mathrm{H}$ ), 6.86 (d, $J=7.6 \mathrm{~Hz}, 2 \mathrm{H}$ ), $5.84(\mathrm{dt}, J=16.8,9.2 \mathrm{~Hz}, 1 \mathrm{H}), 5.64$ (dt, $J=$ 16.8, 8.0 Hz, 1H), $5.21(\mathrm{dd}, J=16.4,10.0 \mathrm{~Hz}, 2 \mathrm{H}), 5.01$ (t, $J=10.4 \mathrm{~Hz}, 2 \mathrm{H}), 4.52(\mathrm{~d}$, $J=7.2 \mathrm{~Hz}, 1 \mathrm{H}$ ), 3.79 (s, 3H), 3.07 (q, $J=7.6 \mathrm{~Hz}, 1 \mathrm{H}), 2.16(\mathrm{bs}, 1 \mathrm{H}) ;{ }^{13} \mathrm{C} \mathrm{NMR}$ $\left(\mathrm{CDCl}_{3}, 100 \mathrm{MHz}\right) \delta 159.1,137.1,136.8,134.0,128.1,118.3,117.0,113.6$ (x2), 75.8, 56.3, 55.3; IR ( $\mathrm{NaCl}): 3419$ (br), 1634, $1219 \mathrm{~cm}^{-1}$; HRMS (ESI): $\mathrm{m} / \mathrm{z}[\mathrm{M}+\mathrm{Na}]^{+}$calcd for $\mathrm{C}_{13} \mathrm{H}_{16} \mathrm{O}: 227.1048$, found: 227.1037 .

1(1-Naphthyl)-2-vinylbut-3-en-1-ol (20). The title compound was isolated by column chromatography ( $4: 1$, light petroleum: ethyl acetate, $\left.R_{f}=0.4\right)$ yielding a colorless liquid (192 mg, $0.86 \mathrm{mmol}, 86 \%):{ }^{1} \mathrm{H} \mathrm{NMR}\left(\mathrm{CDCl}_{3}, 400 \mathrm{MHz}\right) \delta 8.08$ (d, $J=$ $8.0 \mathrm{~Hz}, 1 \mathrm{H}), 7.86(\mathrm{~d}, J=8.0 \mathrm{~Hz}, 1 \mathrm{H}), 7.78(\mathrm{~d}, J=8.0 \mathrm{~Hz}, 1 \mathrm{H}), 7.60(\mathrm{~d}, J=7.2 \mathrm{~Hz}$, $1 \mathrm{H}), 7.53-7.45(\mathrm{~m}, 4 \mathrm{H}), 5.96-5.81(\mathrm{~m}, 2 \mathrm{H}), 5.45(\mathrm{dd}, J=6.0,3.6 \mathrm{~Hz}, 1 \mathrm{H}), 5.22(\mathrm{dd}$, $J=10.4,0.8 \mathrm{~Hz}, 1 \mathrm{H}), 5.12-5.03(\mathrm{~m}, 3 \mathrm{H}), 3.41-3.38(\mathrm{~m}, 1 \mathrm{H}), 2.22(\mathrm{~d}, J=3.6 \mathrm{~Hz}$, $1 \mathrm{H}) ;{ }^{13} \mathrm{C} \mathrm{NMR}\left(\mathrm{CDCl}_{3}, 100 \mathrm{MHz}\right) \delta 137.8,137.6,136.3,133.9,130.7,129.1,128.2$, 126.1, 125.5, 125.3, 124.5, 123.3, 118.6, 116.8, 72.9, 54.8; IR (NaCl): 3583, 3084, 1677, 1421, $1265 \mathrm{~cm}^{-1}$; HRMS (ESI): $\mathrm{m} / \mathrm{z}[\mathrm{M}+\mathrm{Na}]^{+}$calcd for $\mathrm{C}_{16} \mathrm{H}_{16} \mathrm{O}: 247.1099$, found: 247.1083 .

2-Phenylpent-4-en-2-ol (21a). ${ }^{8 \mathrm{~d}}$ The title compound was isolated by column chromatography ( $4: 1$, light petroleum: ethyl acetate, $\left.R_{f}=0.4\right)$ yielding a colorless liquid (142 mg, $0.76 \mathrm{mmol}, 76 \%):{ }^{1} \mathrm{H}$ NMR $\left(\mathrm{CDCl}_{3}, 400 \mathrm{MHz}\right) \delta 7.44-7.41(\mathrm{~m}, 2 \mathrm{H})$, 7.38 - $7.33(\mathrm{~m}, 2 \mathrm{H}), 7.28-7.24(\mathrm{~m}, 1 \mathrm{H}), 5.81$ (ddd, $J=17.2,10.4,8.8 \mathrm{~Hz}, 1 \mathrm{H}), 5.74$ (ddd, $J=17.6,10.4,7.2 \mathrm{~Hz}, 1 \mathrm{H}$ ), 5.20 (ddd, $J=10.4,1.6,0.4 \mathrm{~Hz}, 1 \mathrm{H}$ ), $5.16-5.13$ $(\mathrm{m}, 1 \mathrm{H}), 5.14-5.09(\mathrm{~m}, 2 \mathrm{H}), 3.15(\mathrm{t}, J=7.6 \mathrm{~Hz}, 1 \mathrm{H}), 2.17(\mathrm{~s}, 1 \mathrm{H}), 1.57(\mathrm{~s}, 3 \mathrm{H}) ;{ }^{13} \mathrm{C}$ $\mathrm{NMR}\left(\mathrm{CDCl}_{3}, 100 \mathrm{MHz}\right) \delta 146.4,136.8,136.3,127.9,126.7,125.5,118.1,75.3,29.8$, 27.6; HRMS (ESI): $\mathrm{m} / z$ [M+Na] calcd for $\mathrm{C}_{13} \mathrm{H}_{16} \mathrm{O}: 211.1099$, found: 211.1087 . 
1-(Cinnamyl)-2-vinylbut-3-en-1-ol (22). ${ }^{6 \mathrm{~b}}$ The title compound was isolated by column chromatography $\left(4: 1\right.$, light petroleum: ethyl acetate, $\left.R_{f}=0.3\right)$ yielding a dark yellow liquid (120 mg, $0.60 \mathrm{mmol}, 60 \%):{ }^{1} \mathrm{H} \mathrm{NMR}\left(\mathrm{CDCl}_{3}, 400 \mathrm{MHz}\right) \delta 7.46-7.41(\mathrm{~m}$, $2 \mathrm{H}), 7.38-7.34(\mathrm{~m}, 2 \mathrm{H}), 7.31-7.26(\mathrm{~m}, 1 \mathrm{H}), 6.65(\mathrm{dd}, J=16.0,0.8 \mathrm{~Hz}, 1 \mathrm{H}), 6.27$ $(\mathrm{dd}, J=16.0,6.4 \mathrm{~Hz}, 1 \mathrm{H}), 5.97-5.88(\mathrm{~m}, 1 \mathrm{H}), 5.92-5.86(\mathrm{~m}, 1 \mathrm{H}), 5.29-5.19(\mathrm{~m}$, $4 \mathrm{H}), 4.30(\mathrm{t}, J=6.0 \mathrm{~Hz}, 1 \mathrm{H}), 3.08-3.02(\mathrm{tq}, J=7.2,1.2 \mathrm{~Hz}, 1 \mathrm{H}), 2.12(\mathrm{bs}, 1 \mathrm{H}) ;{ }^{13} \mathrm{C}$ $\mathrm{NMR}\left(\mathrm{CDCl}_{3}, 100 \mathrm{MHz}\right) \delta 136.8,136.6,131.6,129.7,128.6,127.7,126.6,118.0$, 117.7, 76.8, 55.0; IR (NaCl): 3400, 3061, $1636 \mathrm{~cm}^{-1}$; HRMS (ESI): $\mathrm{m} / \mathrm{z}[\mathrm{M}+\mathrm{H}]^{+}$calcd for $\mathrm{C}_{14} \mathrm{H}_{16} \mathrm{O}: 201.1279$, found: 201.1273 .

1-Cyclohexyl-2-vinylbut-3-en-1-ol (23). ${ }^{8 \mathrm{~d}}$ The title compound was isolated by column chromatography ( $4: 1$, light petroleum: ethyl acetate, $\left.R_{f}=0.5\right)$ yielding a colorless liquid (137 mg, $0.76 \mathrm{mmol}, 76 \%):{ }^{1} \mathrm{H} \mathrm{NMR}\left(\mathrm{CDCl}_{3}, 400 \mathrm{MHz}\right) \delta 5.85$ (ddd, J $=16.4,10.0,8.4 \mathrm{~Hz}, 1 \mathrm{H}), 5.85-5.77(\mathrm{~m}, 1 \mathrm{H}), 5.17(\mathrm{dd}, J=10.0,1.6 \mathrm{~Hz}, 1 \mathrm{H}), 5.12$ (ddd, $J=10.8,2.0,1.2 \mathrm{~Hz}, 1 \mathrm{H}), 5.12-5.10(\mathrm{~m}, 1 \mathrm{H}), 5.09-5.07(\mathrm{~m}, 1 \mathrm{H}), 3.29-3.25$ $(\mathrm{m}, 1 \mathrm{H}), 2.95(\mathrm{q}, J=7.2 \mathrm{~Hz}, 1 \mathrm{H}), 1,88-1.79(\mathrm{~m}, 1 \mathrm{H}), 1.78-1.69(\mathrm{~m}, 2 \mathrm{H}), 1.68-1.57$ $(\mathrm{m}, 2 \mathrm{H}), 1.48-1.37(\mathrm{~m}, 1 \mathrm{H}), 1.29-1.00(\mathrm{~m}, 5 \mathrm{H}) ;{ }^{13} \mathrm{C} \mathrm{NMR}\left(\mathrm{CDCl}_{3}, 100 \mathrm{MHz}\right) \delta$ 136.5, 135.3, 115.4, 114.2, 75.3, 49.7, 38.4, 27.9, 24.9, 24.5, 24.4, 24.2; IR (NaCl): 3435 (br), 3077, $1633 \mathrm{~cm}^{-1}$; HRMS (ESI): $\mathrm{m} / \mathrm{z}[\mathrm{M}+\mathrm{Na}]^{+}$calcd for $\mathrm{C}_{12} \mathrm{H}_{20} \mathrm{O}: 203.1412$, found: 203.1406 .

6-Methyl-3-vinylhept-1-en-4-ol (24). ${ }^{18}$ The title compound was isolated by column chromatography $\left(4: 1\right.$, light petroleum: ethyl acetate, $\left.R_{f}=0.4\right)$ yielding a colorless liquid (106 mg, $0.69 \mathrm{mmol}, 69 \%):{ }^{1} \mathrm{H} \mathrm{NMR}\left(\mathrm{CDCl}_{3}, 400 \mathrm{MHz}\right) \delta 5.86$ (ddd, $J=10.0$, 8.0, $4.8 \mathrm{~Hz}, 1 \mathrm{H}$ ), 5.81 (ddd, $J=12.8,7.6,4.4 \mathrm{~Hz}, 1 \mathrm{H}$ ), 5.21 (dd, $J=10.4,1.2 \mathrm{~Hz}$, $1 \mathrm{H}), 5.18-5.15(\mathrm{~m}, 1 \mathrm{H}), 5.16-5.14(\mathrm{~m} .1 \mathrm{H}), 5.13(\mathrm{ddd}, J=5.6,2.4,0.8 \mathrm{~Hz}, 1 \mathrm{H})$, $3.65(\mathrm{dt}, J=9.6,4.4 \mathrm{~Hz}, 1 \mathrm{H}), 2.79(\mathrm{q}, J=7.6 \mathrm{~Hz}, 1 \mathrm{H}), 1.89-1.79(\mathrm{~m}, 1 \mathrm{H}), 1.65(\mathrm{~d}, J$ $=4.4 \mathrm{~Hz}, 1 \mathrm{H}), 1.36-1.29(\mathrm{~m}, 2 \mathrm{H}), 0.95(\mathrm{~d}, J=6.8 \mathrm{~Hz}, 3 \mathrm{H}), 0.92(\mathrm{~d}, J=6.4 \mathrm{~Hz}, 3 \mathrm{H})$; ${ }^{13} \mathrm{C} \mathrm{NMR}\left(\mathrm{CDCl}_{3}, 100 \mathrm{MHz}\right) \delta 137.6,136.9,117.6,116.9,71.2,55.4,43.4,24.6,23.8$, 21.7; IR (NaCl): 3368 (br), 3079, $1635 \mathrm{~cm}^{-1}$; HRMS (ESI): $\mathrm{m} / \mathrm{z}[\mathrm{M}+\mathrm{H}]^{+}$calcd for $\mathrm{C}_{10} \mathrm{H}_{18} \mathrm{O}: 155.1436$, found: 155.1429 .

3-Vinylpent-4-ene-1,2-diol (25). The title compound was isolated by column chromatography $\left(4: 1\right.$, light petroleum: ethyl acetate, $\left.R_{f}=0.2\right)$ yielding a colorless oil (92 mg, 0.72 mmol, 72\%): ${ }^{1} \mathrm{H}$ NMR $\left(\mathrm{CDCl}_{3}, 400 \mathrm{MHz}\right) \delta 5.86$ (ddd, $J=16.8 .10 .8,8.0$ 
$\mathrm{Hz}, 1 \mathrm{H}), 5.77$ (ddd, $J=16.8,10.8,8.0 \mathrm{~Hz}, 1 \mathrm{H}), 5.21-5.16(\mathrm{~m}, 2 \mathrm{H}), 5.15-5.11(\mathrm{~m}$, $2 \mathrm{H}), 3.71-3.62(\mathrm{~m}, 2 \mathrm{H}), 3.55-3.47(\mathrm{~m}, 1 \mathrm{H}), 3.23(\mathrm{bs}, 1 \mathrm{H}), 3.15(\mathrm{~m}, 1 \mathrm{H}), 2.88(\mathrm{q}, J=$ 8.0 Hz, 1H); ${ }^{13} \mathrm{C}$ NMR $\left(\mathrm{CDCl}_{3}, 100 \mathrm{MHz}\right) \delta 136.8,136.7,117.5,117.1,73.6,64.6$, 51.7; IR (NaCl): 3365 (br), 3003, $1637 \mathrm{~cm}^{-1}$; HRMS (ESI): $\mathrm{m} / \mathrm{z}[\mathrm{M}+\mathrm{Na}]^{+}$calcd for $\mathrm{C}_{7} \mathrm{H}_{12} \mathrm{O}_{2}: 151.0735$, found: 151.0729 .

1-(Trimethylsilyl)-4-vinylhex-5-en-1-yn-3-ol (14). Aldehyde 12 (1.51 g, 12.00 $\mathrm{mmol}$ ) and (E)-5-bromopenta-1,3-diene 13 (2.12 g, $14.40 \mathrm{mmol})$ were added to absolute ethanol $(20 \mathrm{~mL})$ and allowed to stir at room temperature. Indium powder ( $2.05 \mathrm{~g}, 18.00 \mathrm{mmol})$ was added in portions, so as not to increase the temperature of the reaction mixture, and then allowed to stir at room temperature for $72 \mathrm{~h}$. The reaction mixture was diluted with diethyl ether and then filtered through a plug of silica. The solvent was then removed by evaporation under reduced pressure and the crude product purified by column chromatography. The title compound was isolated by column chromatography $\left(4: 1\right.$, light petroleum: ethyl acetate, $\left.R_{f}=0.6\right)$ yielding a pale yellow oil $(1.87 \mathrm{~g}, 9.60 \mathrm{mmol}, 80 \%):{ }^{1} \mathrm{H} \mathrm{NMR}\left(\mathrm{CDCl}_{3}, 400 \mathrm{MHz}\right) \delta 5.91$ (ddd, $J=17.2,10.4,8.0 \mathrm{~Hz}, 2 \mathrm{H}), 5.28-5.18(\mathrm{~m}, 4 \mathrm{H}), 4.33(\mathrm{dd}, J=7.2,5.6 \mathrm{~Hz}, 1 \mathrm{H})$, 3.07 - $3.02(\mathrm{~m}, 1 \mathrm{H}), 2.10(\mathrm{~d}, J=7.2 \mathrm{~Hz}, 1 \mathrm{H}), 0.19(\mathrm{~s}, 9 \mathrm{H}) ;{ }^{13} \mathrm{C} \mathrm{NMR}\left(\mathrm{CDCl}_{3}\right.$, $100 \mathrm{MHz}) \delta 136.1,135.7,118.7,118.2,104.6,91.2,65.3,54.7,0.01$; IR (NaCl): 3429 (br), 3008, 2173, $1638 \mathrm{~cm}^{-1}$; HRMS (ESI): $\mathrm{m} / \mathrm{z}[\mathrm{M}+\mathrm{Na}]^{+}$calcd for $\mathrm{C}_{11} \mathrm{H}_{18} \mathrm{OSi}$ : 217.1019, found: 217.1020 .

\section{General procedure for the rearrangement from branched to straight chain diene}

Branched chain diene $(1.0 \mathrm{mmol})$ in dichloromethane $(2.0 \mathrm{~mL} / \mathrm{mmol})$ was added to indium trifluoromethanesulfonate $(10 \mathrm{~mol} \%)$ and the aldehyde $(10 \mathrm{~mol} \%)$ and allowed to stir at room temperature for $24 \mathrm{~h}$. The reaction mixture was then transferred to a separating funnel, diluted with further dichloromethane and washed with distilled water. The organic extracts were retained, dried $\left(\mathrm{Na}_{2} \mathrm{SO}_{4}\right)$, filtered and the solvent removed by evaporation under reduced pressure. The title compound was isolated by column chromatography. The following compounds were synthesized using this method. 
(E)-1-Phenylhexa-3,5-dien-1-ol (27). The title compound was isolated by column chromatography $\left(4: 1\right.$, light petroleum: ethyl acetate, $\left.R_{f}=0.3\right)$ yielding a colorless liquid (116 mg, $0.67 \mathrm{mmol}, 67 \%):{ }^{1} \mathrm{H}$ NMR (400MHz, $\left.\mathrm{CDCl}_{3}\right) \delta 7.42$ - $7.29(\mathrm{~m}, 5 \mathrm{H})$, 6.35 (td, $J=16.8,10.0 \mathrm{~Hz}, 1 \mathrm{H}), 6.22(\mathrm{dd}, J=15.2,10.4 \mathrm{~Hz}, 1 \mathrm{H}), 5.71$ (dt, $J=15.2$, $7.2 \mathrm{~Hz}, 1 \mathrm{H}), 5.18$ (d, J = $16.4 \mathrm{~Hz}, 1 \mathrm{H}), 5.06$ (d, $J=10.0 \mathrm{~Hz}, 1 \mathrm{H}), 4.80-4.74(\mathrm{~m}, 1 \mathrm{H})$, 2.60 - $2.56(\mathrm{~m}, 2 \mathrm{H}), 2.13(\mathrm{~d}, J=3.2 \mathrm{~Hz}, 1 \mathrm{H}) ;{ }^{13} \mathrm{C}$ NMR $\left(100 \mathrm{MHz}, \mathrm{CDCl}_{3}\right) \delta 143.9$, 136.8, 134.4, 130.1, 128.5, 127.7, 125.8, 116.3, 73.7, 42.6; IR ( $\mathrm{NaCl})$ : 3411, 3080, 3030, 1634, $1603 \mathrm{~cm}^{-1}$; HRMS (ESI): $\mathrm{m} / \mathrm{z}\left[\mathrm{M}-\mathrm{H}^{+}\right]$calcd for $\mathrm{C}_{12} \mathrm{H}_{13} \mathrm{O}: 173.0966$, found: 173.0961.

(E)-1-(2-Bromophenyl)hexa-3,5-dien-1-ol (28). The title compound was isolated by column chromatography ( $4: 1$, light petroleum: ethyl acetate, $\left.R_{f}=0.3\right)$ yielding a colorless liquid $182 \mathrm{mg}, 0.72 \mathrm{mmol}, 71 \%):{ }^{1} \mathrm{H}$ NMR $\left(400 \mathrm{MHz}, \mathrm{CDCl}_{3}\right) \delta 7.82$ (d, $\mathrm{J}=$ $7.6 \mathrm{~Hz}, 1 \mathrm{H}), 7.64(\mathrm{dd}, J=8.0,1.2 \mathrm{~Hz}, 1 \mathrm{H}), 7.18-7.08(\mathrm{~m}, 2 \mathrm{H}), 6.07(\mathrm{~d}, J=1.2 \mathrm{~Hz}$, $1 \mathrm{H}), 5.95-5.84(\mathrm{~m}, 1 \mathrm{H}), 5.71$ (dd, $J=16.0,5.6 \mathrm{~Hz}, 1 \mathrm{H}), 5.29$ (dd, $J=5.6,2.4 \mathrm{~Hz}$, $1 \mathrm{H}), 4.58(\mathrm{dd}, J=10.8,5.6 \mathrm{~Hz}, 1 \mathrm{H}), 2.64-2.53(\mathrm{~m}, 1 \mathrm{H}), 2.39-2.28(\mathrm{~m}, 1 \mathrm{H}) ;{ }^{13} \mathrm{C}$ NMR $\left(100 \mathrm{MHz}, \mathrm{CDCl}_{3}\right) \delta 142.7,133.1,132.7,132.6,130.5,128.5,121.2,100.6$, 78.0, 77.2, 72.1, 40.6; IR (NaCl) 3428, 3077, 1636, $619 \mathrm{~cm}^{-1}$; HRMS (ESI): $\mathrm{m} / \mathrm{z}$ [M$\mathrm{H}^{+}$] calcd for $\mathrm{C}_{12} \mathrm{H}_{12} \mathrm{O}^{79} \mathrm{Br}$ : 251.0072, found: 251.0065 .

(E)-1-(3-Bromophenyl)hexa-3,5-dien-1-ol (29). The title compound was isolated by column chromatography (4:1, light petroleum: ethyl acetate, $\left.R_{f}=0.3\right)$ yielding a colorless liquid (175 mg, $0.69 \mathrm{mmol}, 69 \%):{ }^{1} \mathrm{H}$ NMR $\left(400 \mathrm{MHz}, \mathrm{CDCl}_{3}\right) \delta 7.57$ - 7.54 $(\mathrm{m}, 1 \mathrm{H}), 7.45-7.41(\mathrm{~m}, 1 \mathrm{H}), 7.31-7.22(\mathrm{~m}, 2 \mathrm{H}), 6.34(\mathrm{td}, J=16.8,10.0 \mathrm{~Hz}, 1 \mathrm{H})$, $6.20(\mathrm{dd}, J=15.2,10.4 \mathrm{~Hz}, 1 \mathrm{H}), 5.68$ (dt, $J=15.2,7.6 \mathrm{~Hz}, 1 \mathrm{H}), 5.19(\mathrm{~d}, J=16.8 \mathrm{~Hz}$, $1 \mathrm{H}), 5.08(\mathrm{~d}, J=10.0 \mathrm{~Hz}, 1 \mathrm{H}), 4.75-4.71(\mathrm{~m}, 1 \mathrm{H}), 2.59-2.46(\mathrm{~m}, 2 \mathrm{H}), 2.11(\mathrm{~d}, J=$ $3.2 \mathrm{~Hz}, 1 \mathrm{H}) ;{ }^{13} \mathrm{C}$ NMR $\left(100 \mathrm{MHz}, \mathrm{CDCl}_{3}\right) \delta 146.2,136.6,134.9,132.8,130.6,130.0$, 129.4, 124.4, 122.6, 116.7, 72.9, 42.7; IR (NaCl) 3391, 3084, 1637, $704 \mathrm{~cm}^{-1}$; HRMS (ESI): $m / z\left[M-\mathrm{H}^{+}\right]$calcd for $\mathrm{C}_{12} \mathrm{H}_{12} \mathrm{O}^{79} \mathrm{Br}: 251.0072$, found: 251.0059 .

(E)-1-(4-Bromophenyl)hexa-3,5-dien-1-ol (30). The title compound was isolated by column chromatography ( $4: 1$, light petroleum: ethyl acetate, $\left.R_{f}=0.3\right)$ yielding a colorless liquid (180 mg, $0.71 \mathrm{mmol}, 72 \%):{ }^{1} \mathrm{H}$ NMR $\left(400 \mathrm{MHz}, \mathrm{CDCl}_{3}\right) \delta 7.46$ (d, J = $8.0 \mathrm{~Hz}, 2 \mathrm{H}), 7.22(\mathrm{~d}, J=8.4 \mathrm{~Hz}, 2 \mathrm{H}), 6.30(\mathrm{td}, J=16.8,10.8 \mathrm{~Hz}, 1 \mathrm{H}), 6.15(\mathrm{dd}, J=$ 15.6, 10.4 Hz, 1H), 5.63 (dt, $J=14.4,8.0 \mathrm{~Hz}, 1 \mathrm{H}), 5.15$ (dd, $J=16.8,7.6 \mathrm{~Hz}, 1 \mathrm{H}$ ), 
5.07 - $5.01(\mathrm{~m}, 1 \mathrm{H}), 4.74$ - $4.66(\mathrm{~m}, 1 \mathrm{H}), 2.55$ - $2.43(\mathrm{~m}, 2 \mathrm{H}), 2.00(\mathrm{~d}, J=3.2 \mathrm{~Hz}, 1 \mathrm{H})$; ${ }^{13} \mathrm{C} \mathrm{NMR}\left(100 \mathrm{MHz}, \mathrm{CDCl}_{3}\right) \delta$ 142.8. 136.6, 134.9, 131.6, 129.4, 127.6 (x2), 121.4, 116.6, 70.0, 42.7; IR (NaCl) 3419, 3079, 1635, $623 \mathrm{~cm}^{-1}$; HRMS (ESI): m/z [M-H $\left.{ }^{+}\right]$ calcd for $\mathrm{C}_{12} \mathrm{H}_{12} \mathrm{O}^{79} \mathrm{Br}$ : 251.0072, found: 251.0066 .

(E)-1-(1-Naphthyl)hexa-3,5-dien-1-ol (14) (32). The title compound was isolated by column chromatography (4:1, light petroleum: ethyl acetate, $\left.R_{f}=0.3\right)$ yielding a colorless liquid (152 mg, $0.68 \mathrm{mmol}, 72 \%):{ }^{1} \mathrm{H}$ NMR $\left(400 \mathrm{MHz}, \mathrm{CDCl}_{3}\right) \delta 8.06$ (d, J = $8.8 \mathrm{~Hz}, 1 \mathrm{H}), 7.88-7.86(\mathrm{~m}, 1 \mathrm{H}), 7.78(\mathrm{~d}, J=7.6 \mathrm{~Hz}, 1 \mathrm{H}), 7.66(\mathrm{~d}, J=7.2 \mathrm{~Hz}, 1 \mathrm{H})$, 7.65 - $7.46(\mathrm{~m}, 3 \mathrm{H}), 6.34$ (dt, $J=16.8,10.4 \mathrm{~Hz}, 1 \mathrm{H}), 6.22(\mathrm{dd}, J=14.8,10.4 \mathrm{~Hz}, 1 \mathrm{H})$, 5.81 (pent, $J=7.6 \mathrm{~Hz}, 1 \mathrm{H}), 5.52(\mathrm{dd}, J=8.4,3.2 \mathrm{~Hz}, 1 \mathrm{H}), 5.19-5.16(\mathrm{~m}, 1 \mathrm{H}), 5.08$ $5.04(\mathrm{~m}, 1 \mathrm{H}), 2.84-2.75(\mathrm{~m}, 1 \mathrm{H}), 2.66-2.59(\mathrm{~m}, 1 \mathrm{H}), 2.13$ (bs, $1 \mathrm{H}) ;{ }^{13} \mathrm{C} N M R$ $\left(100 \mathrm{MHz}, \mathrm{CDCl}_{3}\right) \delta 139.5,136.8,134.4,133.9,130.5,130.3,129.1,128.1,126.2$, 125.6, 125.6, 123.0, 122.9, 116.4, 70.5, 41.8; HRMS (ESI) $\mathrm{m} / \mathrm{z}\left[\mathrm{M}+\mathrm{Na}^{+}\right]$calcd for $\mathrm{C}_{16} \mathrm{H}_{16} \mathrm{O}: 247.1099$, found: 247.1083.

(E)-1-Cyclohexylhexa-3,5-dien-1-ol (34). The title compound was isolated by column chromatography ( $4: 1$, light petroleum: ethyl acetate, $\left.R_{f}=0.4\right)$ yielding a colorless liquid (108 mg, $0.60 \mathrm{mmol}, 60 \%):{ }^{1} \mathrm{H}$ NMR $\left(400 \mathrm{MHz}, \mathrm{CDCl}_{3}\right) \delta 6.31$ (td, $\mathrm{J}=$ 17.2, $10.4 \mathrm{~Hz}, 1 \mathrm{H}), 6.18$ - $6.08(\mathrm{~m}, 1 \mathrm{H}), 5.70$ (q, J = 8.0 Hz, 1H), 5.11 (d, J = $16.4 \mathrm{~Hz}$, $2 \mathrm{H}), 3.42$ - $3.34(\mathrm{~m}, 1 \mathrm{H}), 2.38$ - $2.29(\mathrm{~m}, 2 \mathrm{H}), 2.22$ - $2.13(\mathrm{~m}, 1 \mathrm{H}), 1.40-0.98(\mathrm{~m}$, $10 \mathrm{H}) ;{ }^{13} \mathrm{C}$ NMR $\left(100 \mathrm{MHz}, \mathrm{CDCl}_{3}\right) \delta 136.9,134.0,131.3,115.8,75.3,41.4,29.9$, 29.7, 26.6, 26.4, 26.3, 22.7; IR (NaCl) 3457, 3088, 2928, $1650 \mathrm{~cm}^{-1}$; HRMS (ESI) $\mathrm{m} / \mathrm{z}\left[\mathrm{M}-\mathrm{H}^{+}\right]$calcd for $\mathrm{C}_{12} \mathrm{H}_{19} \mathrm{O}: 179.1436$, found: 179.1429 .

(E)-1-Isovalerylhexa-3,5-dien-1-ol (35). The title compound was isolated by column chromatography $\left(4: 1\right.$, light petroleum: ethyl acetate, $\left.R_{f}=0.4\right)$ yielding a colorless liquid (97 mg, $0.63 \mathrm{mmol}, 63 \%):{ }^{1} \mathrm{H}$ NMR (400MHz, $\mathrm{CDCl}_{3}$ ) $\delta 6.31$ (dt, $J=16.8,10.4$ $\mathrm{Hz}, 1 \mathrm{H}$ ), 6.12 (dd, $J=15.2,10.8 \mathrm{~Hz}, 1 \mathrm{H}$ ), 5.70 (pent, $J=7.6 \mathrm{~Hz}, 1 \mathrm{H}), 5.13$ (d, $J=$ $12.4 \mathrm{~Hz}, 1 \mathrm{H}), 5.00$ (dd, J = 8.8, $1.2 \mathrm{~Hz}, 1 \mathrm{H}), 3.72-3.68(\mathrm{~m}, 1 \mathrm{H}), 2.36-2.25(\mathrm{~m}, 1 \mathrm{H})$, 2.19 - $2.11(\mathrm{~m}, 1 \mathrm{H}), 1.86-1.72(\mathrm{~m}, 1 \mathrm{H}), 1.49$ (bs, $1 \mathrm{H}), 1.42$ - $1.36(\mathrm{~m}, 1 \mathrm{H}), 1.27$ $1.19(\mathrm{~m}, 1 \mathrm{H}), 0.91-0.88(\mathrm{~m}, 6 \mathrm{H}) ;{ }^{13} \mathrm{C} \mathrm{NMR}\left(100 \mathrm{MHz}, \mathrm{CDCl}_{3}\right) \delta$ 136.9, 134.3, 130.7, 116.0, 69.2, 46.2, 41.3, 24.7, 26.5, 22.1; IR (NaCl) 3584, 3086, 2927, 1651, 1467, 1367, $1004 \mathrm{~cm}^{-1}$; HRMS (ESI) $\mathrm{m} / \mathrm{z}\left[\mathrm{M}+\mathrm{Na}^{+}\right]$calcd for $\mathrm{C}_{10} \mathrm{H}_{18} \mathrm{O}: 177.1255$, found: 177.1241. 
(E)-1-(Trimethylsilyl)octa-5,7-dien-1-yn-3-ol (36). The title compound was isolated by column chromatography ( $4: 1$, light petroleum: ethyl acetate, $\left.R_{f}=0.5\right)$ yielding a pale yellow liquid (110 mg, $0.53 \mathrm{mmol}, 53 \%):{ }^{1} \mathrm{H}$ NMR $\left(400 \mathrm{MHz}, \mathrm{CDCl}_{3}\right) \delta 6.37$ (td, J $=16.8,10.8 \mathrm{~Hz}, 1 \mathrm{H}), 6.26-6.19(\mathrm{~m}, 1 \mathrm{H}), 5.77(\mathrm{td}, J=14.8,7.6 \mathrm{~Hz}, 1 \mathrm{H}), 5.19(\mathrm{dd}, J$ $=16.8,0.8 \mathrm{~Hz}, 1 \mathrm{H}), 5.09$ (dd, $J=10.4,1.2 \mathrm{~Hz}, 1 \mathrm{H}), 4.43(\mathrm{q}, J=6.0 \mathrm{~Hz}, 1 \mathrm{H}), 2.52(\mathrm{t}$, $J=6.4 \mathrm{~Hz}, 2 \mathrm{H}), 0.18(\mathrm{~s}, 9 \mathrm{H}) ;{ }^{13} \mathrm{C}$ NMR $\left(100 \mathrm{MHz}, \mathrm{CDCl}_{3}\right) \delta 137.0,135.0,128.5$, 116.5, 105.9, 90.0, 62.2, 40.9, 0.1; IR (NaCl) 3400, 2999, 2152, $1618 \mathrm{~cm}^{-1}$; HRMS (ESI) $m / z\left[M+H^{+}\right]$calcd for $\mathrm{C}_{11} \mathrm{H}_{18} \mathrm{OSi}: 195.1205$, found: 195.1200 .

\section{Ethyl (E)-3-([1-phenyl-2-vinylbut-3-en-1-yl]oxy)acrylate (38)}

To a solution of diene 15 (192 mg, $1.10 \mathrm{mmol})$ THF (3.00 mL) was added DABCO (12 $\mathrm{mg}, 0.10 \mathrm{mmol}$ ) and the resulting solution stirred under $\mathrm{N}_{2}$ and cooled to $0^{\circ} \mathrm{C}$. To this was added ethyl propiolate $(0.10 \mathrm{~mL}, 1.00 \mathrm{mmol})$ drop wise over $5 \mathrm{~min}$. and the reaction mixture then allowed to warm to room temperature and stirred for overnight. The reaction mixture was then concentrated in vacuo and the resultant oil purified by column chromatography ( $4: 1$, light petroleum: ethyl acetate, $\left.R_{f}=0.8\right)$ yielding a colourless liquid (284 mg, $0.95 \mathrm{mmol}, 95 \%):{ }^{1} \mathrm{H}$ NMR $\left(\mathrm{CDCl}_{3}, 400 \mathrm{MHz}\right) \delta 7.48$ (d, J = $12.8 \mathrm{~Hz}, 1 \mathrm{H}$ ), 7.35 - 7.25 (m, 3H), 7.22 - 7.18 (m, 2H), 5.80 (ddd, J = 17.2, 10.4, 7.6 $\mathrm{Hz}, 1 \mathrm{H}), 5.69$ (ddd, $J=17.2,10.0,7.2 \mathrm{~Hz}, 1 \mathrm{H}), 5.18$ (d, $J=12.4 \mathrm{~Hz}, 1 \mathrm{H}), 5.15-5.04$ $(\mathrm{m}, 2 \mathrm{H}), 5.05-4.98(\mathrm{~m}, 2 \mathrm{H}), 4.83(\mathrm{~d}, \mathrm{~J}=6.0 \mathrm{~Hz}, 1 \mathrm{H}), 4.14-4.09(\mathrm{~m}, 2 \mathrm{H}), 3.24-3.18$ $(\mathrm{m}, 1 \mathrm{H}), 1.20(\mathrm{t}, J=6.8 \mathrm{~Hz}, 3 \mathrm{H}) ;{ }^{13} \mathrm{C} N M R\left(\mathrm{CDCl}_{3}, 100 \mathrm{MHz}\right) \delta 167.8,161.5,137.6$, 136.0, 135.6, 128.4, 128.3, 127.0, 118.0, 117.7, 98.7, 86.4, 59.8, 54.2, 14.4; IR (NaCl): 3584, 2981, 1731, 1651, $1373 \mathrm{~cm}^{-1}$; HRMS (ESI): $\mathrm{m} / \mathrm{z}[\mathrm{M}+\mathrm{Na}]^{+}$calcd for $\mathrm{C}_{17} \mathrm{H}_{20} \mathrm{O}_{3}$ : 295.1310, found: 295.1293.

Ethyl (E)-3-hydroxyocta-5,7-dienoate (39). To a solution of 38 (100 mg, 0.37 $\mathrm{mmol})$ in $\mathrm{CH}_{2} \mathrm{Cl}_{2}(2.00 \mathrm{~mL})$ under a $\mathrm{N}_{2}$ atmosphere at $0^{\circ} \mathrm{C}$ was added TFA $(0.03 \mathrm{~mL}$, $0.41 \mathrm{mmol}$ ) and the resultant reaction mixture allowed to warm to room temperature and stirred overnight. After this period $\mathrm{NaHCO}_{3}(2 \mathrm{~mL})$ was added to the reaction mixture, the reaction mixture was then transferred to a separating funnel with additional $\mathrm{CH}_{2} \mathrm{Cl}_{2}(20 \mathrm{~mL})$ and water $(20 \mathrm{~mL})$, the layers separated, and the aqueous layer extracted with a further portion of $\mathrm{CH}_{2} \mathrm{Cl}_{2}(20 \mathrm{~mL})$. The combined organic extracts were then dried $\left(\mathrm{Na}_{2} \mathrm{SO}_{4}\right)$, filtered and the solvent removed under vacuum and the crude oil purified by column chromatography $(4: 1$, light petroleum: ethyl 
acetate, $R_{f}=0.3$ ) yielding a colourless liquid ( $\left.56 \mathrm{mg}, 0.30 \mathrm{mmol}, 82 \%\right):{ }^{1} \mathrm{H}$ NMR $\left(\mathrm{CDCl}_{3}, 400 \mathrm{MHz}\right) \delta 6.30(\mathrm{dt}, J=16.8,10.4 \mathrm{~Hz}, 1 \mathrm{H}), 6.12(\mathrm{dd}, J=15.2,10.8 \mathrm{~Hz}, 1 \mathrm{H})$, 5.69 (pent, $J=7.2 \mathrm{~Hz}, 1 \mathrm{H}$ ), $5.12(\mathrm{~d}, J=16.8 \mathrm{~Hz}, 1 \mathrm{H}), 5.00(\mathrm{~d}, J=9.6 \mathrm{~Hz}, 1 \mathrm{H}), 4.15$ (q, $J=7.6 \mathrm{~Hz}, 1 \mathrm{H}), 4.11-4.04(\mathrm{~m}, 1 \mathrm{H}), 2.98(\mathrm{~d}, J=3.6 \mathrm{~Hz}, 1 \mathrm{H}), 2.50(\mathrm{dd}, J=16.8$, $4.0 \mathrm{~Hz}, 1 \mathrm{H}$ ), 2.40 (dd, $J=16.4,8.8 \mathrm{~Hz}, 1 \mathrm{H}), 2.36-2.23(\mathrm{~m}, 2 \mathrm{H}), 1.25(\mathrm{t}, J=7.2 \mathrm{~Hz}$, $3 \mathrm{H}) ;{ }^{13} \mathrm{C} \mathrm{NMR}\left(\mathrm{CDCl}_{3}, 100 \mathrm{MHz}\right) \delta 171.9,136.8,134.3,129.7,116.3,67.7,60.8$, 40.7, 39.7, 14.3; IR ( $\mathrm{NaCl}): 3583,2983,2253,1719,1374 \mathrm{~cm}^{-1}$; HRMS (ESI): $\mathrm{m} / \mathrm{z}$ $[\mathrm{M}+\mathrm{Na}]^{+}$calcd for $\mathrm{C}_{10} \mathrm{H}_{16} \mathrm{O}_{3}: 207.0997$, found: 207.0983 .

\section{ASSOCIATED CONTENT}

\section{Supporting Information}

The supporting information is available free of charge on the ACS Publications website at

Copies of ${ }^{1} \mathrm{H}$ and ${ }^{13} \mathrm{C}$ spectra of all products (PDF).

\section{AUTHOR INFORMATION}

\section{Corresponding Author(s)}

*E-mail: G.J.Pritchard@Iboro.ac.uk (G.J.P)

*E-mail: M.C.Kimber@lboro.ac.uk (M.C.K)

E-ma

\section{ORCID}

Martin R. Lindley: 0000-0001-7686-9421

Gareth J. Pritchard: 0000-0002-2925-1722

Marc C. Kimber: 0000-0003-2943-1974

\section{Notes}


The authors declare no competing financial interest.

\section{ACKNOWLEDGEMENTS}

We gratefully acknowledge financial support from Loughborough University (studentship for NJC). We also thank Dr. Mark Edgar (Loughborough) for assistance with NMR assignments.

\section{REFERENCES}

(1) Diels-Alder transformations (a) Bendiabdellah, Y.; Rahman, K. M.; Uranchimeg, B.; Nahar, K. S.; Antonow, D.; Shoemaker, R. H.; Melillo, G.; Zinzalla, G.; Thurston, D. E. Tetracycline analogues with a selective inhibitory effect on HIF-1 $1 \alpha$. Med. Chem. Commum. 2014, 5, 923-926; (b) Bendiabdellah, Y.; Villanueva-Margalef, I.; Misale, A.; Nahar, K. S.; Haque, M. R.; Thurston, D. E.; Zinzalla, G. One-pot synthesis of fused-tetracyclic scaffolds employing a Lewis acid promoted domino reaction of naphthoquinones. Synthesis 2011, 2321-2333; (c) Souweha, M. S.; Arab, A.; ApSimon M.; Fallis, A. G. Diene-transmissive cycloadditions: control of monocycloaddition by self-assembly on a Lewis acid. Org. Lett. 2007, 9, 615-618; (d) Kormann, C.; Heinemann, F. W.; Gmeiner, P. A consecutive Diels-Alder approach toward a Tet repressor directed combinatorial library. Tetrahedron 2006, 66, 68996908; (e) Kwon, O.; Park, S. B.; Schreiber, S. L. Skeletal diversity via a branched pathway: efficient synthesis of 29400 discrete compounds and their arraying into stick solutions. J. Am. Chem. Soc. 2002, 124, 13402-13404; (f) Woo, S.; Squires, N.; Fallis, A. G. Indium-mediated Y-pentadienylation of aldehyde and ketones: crossconjugated trienes for diene-transmissive cycloadditions. Org. Lett. 1999, 1, 573575.

(2) Ring closing metathesis transformations (a) Matovic, R.; Bihelovic, F.; GrudenPavlovic, M.; Saicic, R. N. Total synthesis and biological evaluation of atrop-Obenzyl-desmethylabyssomicin C. Org. Biomol. Chem. 2014, 12, 7682-7685; (b) Hartung, J.; Dornan, P. K.; Grubbs, R. H. Enantioselective olefin metathesis with cyclometalated ruthenium complexes. J. Am. Chem. Soc. 2014, 136, 13029-13037; for a related example using the Pauson-Khand reaction see (c) Fujioka, K.; Yokoe, 
H.; Yoshida, M.; Shishido, K. Total synthesis of penostatin B. Org. Lett. 2012, 14, 244-247.

(3) (a) Nokami, J.; Nomiyama, K.; Matsuda, S.; Imai, N.; Kataoka, K. Highly enantioselective alk-2-enylation of aldehydes through an allyl-transfer reaction. Angew. Chem. Int. Ed. 2003, 42, 1273-1276. For an oxy-Cope process using these substrates see (b) Melekhov, A.; Fallis, A. G. Indium mediated y-pentadienylation of conjugated aldehydes: synthons for hydrindanes by an oxy-Cope-cycloaddition strategy. Tetrahedron Lett. 1999, 40, 7867-7870.

(4) For reviews (a) Qu, Q.; Xuan, W.; Fan, G. - H. Roles of resolvins in the resolution of acute inflammation. Cell. Bio. Int. 2015, 39, 3-22; (b) Ji, R. -R.; Xu, Z. -Z.; Strichartz, G.; Serhan, C. N. Emerging roles of resolvins in the resolution of inflammation and pain. Trends Neurosci. 2011, 34, 599-609; (c) Bannenberg, G. L. Resolvins: current understandings and future potential in the control of inflammation. Curr. Opin. Drug Disc. Dev. 2009, 12, 644-658; also (d) Baker, L. A.; Martin, N. R. W.; Kimber, M. C.; Pritchard, G. J.; Lindley, M. R.; Lewis, M. P. Resolvin E1 $\left(R_{v} E_{1}\right)$ attenuates LPS induced inflammation and subsequent atrophy in C2C12 myotubes. J. Cell. Biochem. 2018, 119, 6094-6103.

(5) (a) Seyferth, D.; Pornet, J. J. (2,4-Pentadienyl)trimethylsilane: a useful pentadienylation reagent. J. Org. Chem. 1980, 45, 1721-1722; (b) Seyferth, D.; Pornet, J.; Weinstein, R. M. A useful dienemethylation agent: 2,4pentadienyltrimethylsilane. Organometallics 1982, 1, 1651-1658.

(6) (a) Hosomi, A.; Saito, M.; Sakurai, H. Tetrahedron Lett. Pentadienylsilanes as new reagents of the siteselective dienylmethylation of electrophiles promoted by a Lewis acid. 1980, 21, 3783-3786; for a later report see (b) Kobayashi, S.; Nishio, K. Facile synthesis of both syn and anti homo allylic alcohols from allyl chlorides via organosilicon intermediates. Chem. Lett. 1994, 1773-1776.

(7) Kauffmann, T.; Gaydoul, K. -R. Synthese neuer organoelementsubstituierter pentadiene. Tetrahedron Lett. 1985, 26, 4067-4070. 
(8) (a) Yanagisawa, A.; Nakashima, H.; Nakatsuka, Y.; Ishiba, A.; Yamamoto, H. Asymmetric addition of allylic stannanes to aldehydes catalyzed by a BINAP.Ag(I) complexes. Bull. Chem. Soc. Jpn. 2001, 74, 1129-1137; (b) Nishigaichi, Y.; Hanano, Y.; Takuwa, A. Simultaneous control of regio- and stereochemistries in the reaction between $\alpha$-alkoxyaldehydes and pentadientyltin. Selective preparations of the four regio- and diastereoisomers. Chem. Lett. 1998, 33-34; (c) Yanagisawa, A.; Nakatsuka, Y.; Nakashima, H.; Yamamoto, H. Asymmetric Y-selective pentadienylation of aldehydes catalyzed by BINAP.Ag(I) complex. Synlett 1997, 933935; (d) Nishigaichi, Y.; Fujimoto, M.; Takuwa, A. Y-Selective pentadienylation of aldehydes and ketones with pentadienyltins by the use of $\mathrm{ZnCl}_{2}$. Synlett 1994, 731733; (e) Nishigaichi, Y.; Fujimoto, M.; Takuwa, A. Unusual $\varepsilon / Y$-regiospecificity of $(E / Z)$-pentadienyltin in the Lewis acid promoted reaction with an aldehyde. J. Chem. Soc. Perkin Trans. 1, 1992, 2581-2582.

(9) (a) Miginiac-Groizeleau, L.; Miginiac, P.; Prevost, C. Reaction of $\alpha, \beta-\gamma, \delta$ unsaturated organometallic compounds with carbonyl groups. Applications. Bull. Soc. Chim. Fr. 1965, 12, 3560-3565; (b) Jung, M. E.; Nichols, C. J. Highly stereoselective synthesis of trans, trans-4-aryl-2,3-oxetanedimethanols: preparation of oxetanocin A analogues. Tetrahedron Lett. 1996, 37, 7667-7670; (c) Rapodo, L. P.; Bulugahapitiya, V.; Renaud, P. Synthesis of non-racemic 1-hydroxycycloalkene1-carboxylic -acid derivatives of $\alpha, \alpha$-dialkylated glycolate derivatives. Helv. Chimica Acta 2000, 83, 1625-1632.

(10) (a) Okamoto, S.; Sato, F. A highly efficient and practical preparation of 2,4pentadienyltitaniums and their $\mathrm{Y}$-selective addition reaction with aldehydes and ketones. J. Organomet. Chem. 2001, 624, 151-156; (b) Kimura, M.; Shimizu, M; Tanaka, S.; Tamaru, Y. Pd-catalyzed nucleophilic allylic alkylation of aliphatic aldehydes by the use of allyl alcohols. Tetrahedron 2005, 61, 3709-3718.

(11) Zellner, A.; Schlosser, M. Pentadienyltitaniums as versatile intermeduates: regio- and stereoselectivies. Synlett 2001, 1016-1018.

(12) (a) Shen, Z. -L.; Wang, S. -Y.; Chok, Y. -K.; Xu, Y. -H.; Loh, T. -P. Organoindium reagents: the preparation and application in organic synthesis. Chem. Rev. 2013, 113, 271-401; (b) Schneider, U.; Kobayashi, S. Low-oxidation state 
indium-catalyzed C-C bond formation. Acc. Chem. Res. 2012, 45, 1331-1344; (c) Yadav, J. S.; Antony, A.; George, J.; Reddy, B. V. S. Recent developments in indium metal and its salts in organic synthesis. Eur. J. Org. Chem. 2010, 591-605; (d) Augé, J.; Lubin-Germain, N.; Uziel, J. Recent advances in indium-promoted organic reactions. Synthesis 2007, 1739-1764.

(13) Hill, A. N.; Delaney, K. M.; Sullivan, T. R.; Mylod, G.; Kiesow, K. H.; Bowyer, W. J. Heterogenous rate constants for indium mediated allylations: cinnamyl chloride in ethanol/water mixtures. J. Phys. Chem. A 2013, 117, 8826-8835.

(14) For an example of using ethanol in the addition of allylbromide see (a) Shin, J. A.; Cha, J. H.; Pae, A. N.; Choi, K. I.; Koh, H. Y.; Kang, H. -K.; Cho, Y. S. Indiummediated diastereoselective allyation reactions: preparation of tert-a-hydroxy acids. Tetrahedron Lett. 2001, 42, 5489-5492; for addition to aldimines see (b); Vilaivan, T.; Winotapan, C.; Banphavichit, V.; Shinada, T.; Ohfune, Y. Indium-mediated asymmetric Barbier-type allylation of aldimines in alcoholic solvents: synthesis of optically active homoallylic amines. J. Org. Chem. 2005, 70, 3464-3471; (c) Vilaivan, T.; Winotapan, C.; Shinada, T.; Ohfune, Y. Indium mediated Barbier-type allylation of aldimines in alcoholic solvents. Tetrahedron Lett. 2001, 42, 9073-9076.

(15) (a) Loh, T. -P.; Tan, K. -T.; Hu, Q. -Y. The first In(OTf) $)_{3}$-catalyzed conversion of kinetically formed homoallylic alcohols into the thermodynamically preferred regioisomers: application to the synthesis of $22 \alpha$-sterols. Angew. Chem. Int. Ed. 2001, 40, 2921-2922; (b) Sumida, S., -I.; Ohga, M.; Mitani, J.; Nokami, J. New and stereoselective synthesis of 1,4-disubstituted buten-4-ols (homoallylic alcohol $\alpha$ products) from the corresponding $\mathrm{Y}$-isomers (3,4-disubstituted buten-4-ols) via an acid-catalyzed allyl-transfer reaction with aldehydes. J. Am. Chem. Soc. 2000, 122, 1310-1313; (c) Nokami, J.; Anthony, L.; Sumida, S. -I. Chem. Eur. J. 2000, 6, 2909; (d) Loh, T. -P.; Lee C. -L. K.; Tan, T. -K. The first example of enantioselective allyl transfer from a linear homoallylic alcohol to an aldehyde. Org. Lett. 2002, 4, 29852987; (e) Malkov, A. V.; Kabeshov, M. A.; Barłog, M.; Kočovský, P. Enantioselective and catalytic methods for $\alpha$-crotylation of aldehydes with a kinetic self-refinement of stereochemistry. Chem. Eur. J. 2009, 15, 1570-1573. 
(16) The rearranged product observed in entries $3-5$ and 12 can conceivably be explained by a Lewis acid catalyzed retro-ene process, see Ripoli, J. -L.; Vallée, Y. Synthetic applications of the retro-ene reaction. Synthesis 1993, 659-677.

(17) (a) Rychnovsky, S. D.; Marumoto, S.; Jaber, J. J. Role of 2-oxonia Cope rearrangements in Prins cyclisation reactions. Org. Lett. 2001, 3, 3815-3818; (b) For a tandem process Dalgard, J. E.; Rychnvosky, S. D. Oxonia-Cope Prins cyclizations: a facile method for the synthesis of tetrahydropyranones bearing quaternary centers. J. Am. Chem. Soc. 2004, 166, 15662-15663.

(18) Riediker, M.; Duthaler, R. O. Enantioselective allyation of carbonyl compounds with titanium-carbohydrate complexes. Angew. Chem. Int. Ed. 1989, 28, 494-495. 